\title{
Introduction to the first issue of Social Network Analysis and Mining journal
}

\author{
Nasrullah Memon • Reda Alhajj
}

Published online: 13 November 2010

(C) Springer-Verlag 2010

Social networks are gaining popularity swiftly and research on social networks is rapidly growing into wellestablished multidisciplinary area which has attracted the attention of researchers and practitioners from academia, government institutions, and the industry. Hence we realized the need for Social Network Analysis and Mining (SNAM) as the pioneering journal that covers the intersection between social networks and computational sciences. The goal of SNAM is to provide a unique forum for excellent research in all aspects of social networks, from foundations to applications. This will allow for a wide range in readership, including researchers and practitioners from social sciences, computational sciences, biological sciences, medicine, engineering, business, etc. We are happy to see researchers extending the foundations of social networks by integrating new computational techniques; others are adopting the social network model as a powerful technique to tackle different problems in a variety of applications.

\footnotetext{
N. Memon

The Maersk Mc-Kinney Moller Institute, University of Southern Denmark, Campusvej 55, 5230 Odense M, Denmark

e-mail: memon@mmmi.sdu.dk

R. Alhajj ( $\square)$

Department of Computer Science,

University of Calgary,

Calgary, AB T2N 1N4, Canada

e-mail: alhajj@ucalgary.ca; rsalhajj@gmail.com

R. Alhajj

Department of Computer Science,

Global University, Beirut, Lebanon
}

This harmony will lead to a wealth of research outcome that should be realized and recognized; hence SNAM is a timely venue that will serve as the major outlet. It required hard work to establish the board of associate editors that includes leading researchers who have already demonstrated major contributions to the field. Also, it was not an easy task to decide on the papers to include in this first issue. We already have a long queue of accepted papers to appear in the upcoming issues. We are currently working hard to minimize the turnaround time for the reviewing process and to publish accepted papers in the online-first system soon after the acceptance decision is finalized.

It is a great pleasure and honor to include in the first issue of SNAM, five papers written by leading researchers in the field. The first paper is by Alvin W. Wolfe who has been conducting research in the field for close to 50 years. The second paper is by John Scott, and the third by Devan Rosen, George A. Barnett and Jang Hyun Kim. The fourth paper is by Dalhia Mani and David Knoke. The fifth paper is by Robert Hanneman and Christian R. Shelton. We would like to thank all these authors for their wonderful papers that we have selected for the first issue, which we also believe will make this into a valuable source for researchers and practitioners.

Last but not least, we would like to thank the members of our board of associate editors for their time and effort in handling the papers; we also extend our thanks to the reviewers who are doing an amazing job by their timely and comprehensive reviews. Various people from Springer deserve large credit for their help and support in realizing this significant project. We would like to thank Stephen Soehnlen for his support and dedication since the idea was originated 2 years ago. Mhanilet de Leon has been very 
patient in handling our requests and in following up with the submissions. Finally, we would like to thank Philipp Kammerer from the production department for taking care of the accepted papers.
Nasrullah Memon and Reda Alhajj.

Editors-in-Chief.

November 2010.

Springer-Verlag Wien, New York. 